Research Paper

\title{
Serum Immune Proteins in Moderate and Severe Chronic Fatigue Syndrome/Myalgic Encephalomyelitis Patients
}

\author{
Sharni Lee Hardcastle ${ }^{\bowtie}$, Ekua Weba Brenu, Samantha Johnston, Thao Nguyen, Teilah Huth, Sandra \\ Ramos, Donald Staines, Sonya Marshall-Gradisnik
}

National Centre for Neuroimmunology and Emerging Diseases, 9.22, G40 Griffith Health Institute, School of Medical Science, Griffith University, Parklands Drive, 4222, Gold Coast, QLD, Australia.

$\triangle$ Corresponding author: Sharni L. Hardcastle, BBioMedSc (Hons1), National Centre for Neuroimmunology and Emerging Diseases, 9.22, G40 Griffith Health Institute, Griffith University, Parklands Drive, Southport, 4222, Gold Coast, Queensland, Australia. Phone: +61 75678 9283; Email: sharni.hardcastle@hotmail.com

(C) 2015 Ivyspring International Publisher. Reproduction is permitted for personal, noncommercial use, provided that the article is in whole, unmodified, and properly cited. See http://ivyspring.com/terms for terms and conditions.

Received: 2015.04.14; Accepted: 2015.07.07; Published: 2015.09.05

\begin{abstract}
Immunological dysregulation is present in Chronic Fatigue Syndrome/Myalgic Encephalomyelitis (CFS/ME), with recent studies also highlighting the importance of examining symptom severity. This research addressed this relationship between CFS/ME severity subgroups, assessing serum immunoglobulins and serum cytokines in severe and moderate CFS/ME patients. Participants included healthy controls $(n=22)$, moderately $(n=22)$ and severely $(n=19)$ affected CFS/ME patients. The 1994 Fukuda Criteria defined CFS/ME and severity scales confirmed mobile and housebound CFS/ME patients as moderate and severe respectively. IL-1 $\beta$ was significantly reduced in severe compared with moderate CFS/ME patients. IL- 6 was significantly decreased in moderate CFS/ME patients compared with healthy controls and severe CFS/ME patients. RANTES was significantly increased in moderate CFS/ME patients compared to severe CFS/ME patients. Serum IL-7 and IL-8 were significantly higher in the severe CFS/ME group compared with healthy controls and moderate CFS/ME patients. IFN- $\gamma$ was significantly increased in severe CFS/ME patients compared with moderately affected patients. This was the first study to show cytokine variation in moderate and severe CFS/ME patients, with significant differences shown between CFS/ME symptom severity groups. This research suggests that distinguishing severity subgroups in CFS/ME research settings may allow for a more stringent analysis of the heterogeneous and otherwise inconsistent illness.
\end{abstract}

Key words: Cytokine, Immunoglobulin, Chronic Fatigue Syndrome, Serum Protein, Inflammation

\section{Introduction}

Immunological dysregulation can be caused or influenced by changes in serum immune protein levels, which play a key role in living cells by allowing specific interactions with other molecules [1]. Many studies have assessed the levels of cytokines and immunoglobulins in Chronic Fatigue Syndrome/Myalgic Encephalomyelitis (CFS/ME) although even with conflicting and inconsistent results, this may suggest potential shifts in immune regulations in the illness [2].

CFS/ME is a severely debilitating illness which causes patients to experience both physical and cognitive impairment alongside a range of accompanying symptoms [3, 4]. CFS/ME has an unknown aetiology, with diagnosis made in accordance with symptom-specific criteria [3]. While the pathomechanism of CFS/ME is unknown, immunological dysregulation consistently occurs in the illness. However validation is required as inconsistencies are often found between studies. Studies have shown that CFS/ME patients demonstrate reduced natural killer (NK) cell cytotoxic activity [2, 5-9], altered dendritic cell (DC) phenotypes 
[9], decreased $\mathrm{CD}^{+} \mathrm{T}$ cell activity [6] and potential B cell activation [10]. Compromises to innate immune cell functioning can interfere with, or reflect, adaptive processes and translate into shifts in cytokine patterns that are either pro- or anti-inflammatory [11]

Cytokine imbalances in peripheral blood cells may have implications for physiological and psychological functions, with altered cytokine secretions impacting on endothelial, cognitive and cardiovascular body systems [12-14]. There are inconsistent alterations in cytokine patterns in CFS/ME for instance some studies demonstrated elevated IL-1 $\beta$ (interleukin 1 beta), IL-4, IL-5 and IL-6 and reduced IL-8, while other research found no changes to cytokines in the illness $[15,16]$. NK cell dysfunction, namely reduced cytotoxic activity, is consistent in CFS/ME, therefore NK cell derived cytokines, such as IFN- $\gamma$ (interferon gamma), may demonstrate altered immunity patterns and associated cytokine shifts in the illness [2, 15-22].

Cytokine and/or immunoglobulin levels are often altered in diseased states, consequently leading to physiological symptoms [23]. Immunoglobulin levels have also been inconsistently varied in CFS/ME patients [24, 25]. Most studies demonstrate no change to immunoglobulins in CFS/ME although some studies have found low IgM, IgA and IgG levels in CFS/ME patients $[2,16]$. Immunoglobulin levels may also be linked to the reduced NK activity shown in CFS/ME as intravenous immunoglobulin (IVIg) treatments have altered NK cell activity in immune-mediated diseases, such as Kawasaki disease, dermatomyositis and multiple sclerosis (MS). Suppression of NK cell cytotoxic activity is also mediated by the antigen binding portion $\mathrm{F}(\mathrm{ab})_{2}$ of immunoglobulin and hence IVIg infusion also improves antibody-dependent cell-mediated cytotoxicity (ADCC) [26, 27].

A clinically distinct housebound group of CFS/ME patients recently displayed reductions in NK cell cytotoxic activity, increased CD14-CD16 ${ }^{+}$DCs and altered B and iNKT cell phenotypes when compared with moderately affected patients, highlighting the importance of assessing severity subgroups in the illness [9]. In an attempt to control for heterogeneity within a CFS/ME patient cohort, defining CFS/ME patients according to specific clinical characteristics allows for further analysis of the illness [28]. Symptom severity may be related to the extent of immune dysfunction found in CFS/ME patients, placing importance on the recognition of severity subgroups to allow an extensive analysis of the illness. Cytokine imbalances may be related to disease pathologies and therefore should also be assessed in severity subgroups of CFS/ME patients.

This study was the first to examine serum cytokines and immunoglobulins in severe CFS/ME pa- tients in comparison to moderate CFS/ME patients and healthy controls. The purpose of this study was to further examine the relationship between CFS/ME severity subgroups by assessing IgA, IgM, IgG2, IgG4 and IgG Total immunoglobulins and inflammatory cytokines in moderate and severe CFS/ME patients.

\section{Methods}

\section{Ethical Clearance}

The Griffith University Human Research Ethics Committee granted ethical approval for this study after an extensive review (GU Ref No: MSC/23/12/HREC).

\section{Participant Recruitment}

All participants were aged between 20 and 65 years old and were recruited using CFS/ME support groups, social medial, email advertisements and an expression of interest database. All those to be included in the study were briefed and provided written informed consent prior to participating.

All CFS/ME patients included in the research had the illness for at least 6 months prior to the study and were previously diagnosed with CFS/ME by a primary physician. The application of the $1994 \mathrm{Fu}-$ kuda diagnostic criteria was then used in combination with an extensive questionnaire delineating each patient's diagnosis, history and symptoms. A General Practitioner also conducted clinical assessments on all research participants, including patient symptoms, blood pressure, temperature and heart rate. Participants were subjected to stringent exclusion criteria to eliminate confounding co-morbidities or conditions that may influence the immunological data or the participant's illness state. Participants were excluded from the research if they had been diagnosed with an autoimmune, heart or thyroid-related disorder, psychosis, major depression, breast feeding, pregnant, a smoker, if they were taking strong hormone-related medications or if they experienced symptoms of CFS/ME but did not meet the 1994 Fukuda criteria for the illness.

The CFS/ME patients were classified as either mobile or housebound and these severities were translated to 'moderate' and 'severe' subgroups respectively. Moderate CFS/ME patients were those who were mobile, initially identified as those who were able to regularly leave the house unassisted and who had the potential to maintain a job, even with reduced hours. Severe CFS/ME patients were those who were initially identified as housebound, unable to sustain a job due to the constraints of their symptoms and those who were not able to leave the house unassisted. These severity subgroups were then confirmed through the use of an extensive questionnaire 
containing routinely used severity scales in CFS/ME: the Fatigue Severity Scale (FSS), Dr Bell's Disability Scale, the FibroFatigue Scale and the Karnofsky Performance Scale (KPS) [9]. CFS/ME patients who were severely affected by their symptoms were visited in their homes by a team with a mobile qualified phlebotomist and a General Practitioner. Moderate CFS/ME patients and healthy controls participated in the research at a designated collection site where they were met by the General Practitioner and qualified phlebotomist. There were 63 participants included the study, including age and gender matched healthy controls $(n=22)$, moderate CFS/ME $(n=22)$ and severe CFS/ME (n=19) patients.

\section{Sample Preparation}

A morning non-fasting blood sample was collected from the antecubital vein of participants into serum separating tubes (SST). All participants' blood was collected between 8:30am and 11:30am and samples were kept in a cooler box until serum was obtained from centrifugation and snap frozen within 5 hours of the initial collection. Pathology Queensland also conducted an initial full blood count assessment on each participant to ensure that patients were within the ranges for the whole blood cell parameters.

\section{Immunoglobulin Analysis}

Concentrations of the immunoglobulins $\operatorname{IgA}$,

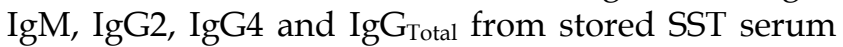
were measured by flow cytometry using a BD CBA Human Immunoglobulin Flex Set system (BD Biosciences, San Diego, CA) as per manufacturer's instructions. FCap Array software (BD Biosciences, San Diego, CA) was then used for the construction of standard curves and for analysis of flow cytometric data.

\section{Cytokine Analysis}

Stored SST serum was thawed and a Bio-Plex Pro human cytokine 27-plex immunoassay kit (Bio-Rad Laboratories Inc, Hercules, CA) was used for the detection of inflammatory cytokines. Cytokines detected in the kit included IL-1 $\beta$, IL-1ra (interleukin 1 receptor agonist), IL-2, IL-4, IL-5, IL-6, IL-7, IL-8, IL-9, IL-10, IL-12p70, IL-13, IL-17, FGF (fibroblast growth factor), eotaxin (CCL11), G-CSF (granulocyte colony-stimulating factor), GM-CSF (granulocyte macrophage colony-stimulating factor), IFN- $\gamma$, IP-10 (interferon gamma-induced protein 10, CXCL10), PDGF-BB (platelet-derived growth factor-BB), RANTES (regulated on activation, normal $\mathrm{T}$ cell expressed and secreted, CCL5), TNF-a (tumor necrosis factor alpha), MCP1 (monocyte chemotactic protein 1), MIP1a (macrophage inflammatory protein alpha), MIP1b (macrophage inflammatory protein beta), and VEGF (vascular endothelial growth factor). The kit used magnetic beads to simultaneously detect cytokine levels and data was obtained in duplicates using the Bio-Plex system in combination with Bio-Plex Manager software (Bio-Rad Laboratories Inc, Hercules, CA).

\section{Data and Statistical Analysis}

Statistical analyses were performed using SPSS statistical software version 22.0. All experimental data represented in this study are reported as plus/minus the standard error of the mean ( + SEM) or plus/minus the standard deviation (SD) as specified. Comparative assessments among the three participant groups (control, moderate CFS/ME and severe CFS/ME patients) were performed using the Kruskal Wallis test of independent variables based on rank sums to determine the magnitude of differences between groups when parameters were not normally distributed. Normally distributed parameters were compared between groups using the analysis of variance test (ANOVA). The Mann-Whitney U or least significant difference (LSD) post hoc tests were used for nonparametric or parametric data respectively to determine significant differences between the groups where $p$ values of statistical significance were set at an alpha criterion at $p<0.05$. Spearman's correlation was conducted on parameters to determine correlates where statistical significance was accepted as $p<0.01$. Outliers were identified using a boxplot technique on SPSS software where extreme outliers were highlighted if they presented beyond the plot's whiskers [29]. Extreme outliers were identified as points beyond an outer fence, defined as the lower quartile - 1.5 $x$ interquartile range (IQ) or the upper quartile plus 3 $x$ IQ. These extreme outliers were then handled by eliminating particular data points from the analysis [29].

\section{Results}

\section{Participants}

Data were available for 63 participants in total, including 22 healthy controls, 22 moderate CFS/ME and 19 severe CFS/ME patients. The mean ( \pm standard deviation) ages for the control, moderate CFS/ME and severe CFS/ME patients were $40.14 \pm 2.38,42.09$ \pm 2.72 and $40.21 \pm 1.57$ respectively. There was no statistical difference in age or gender between participant groups ( $p=0.598,0.324$ respectively) (Table 1$)$. The 1994 Fukuda criteria for CFS/ME were satisfied by all moderate and severe CFS/ME participants.

In all severity scales used, including activity/ability level based on percentage of optimal functioning $(0-100 \%)$, the FibroFatigue Scale, the FSS, the KPS and Dr Bell's Disability Scale, there were statistically significant differences between all participant 
groups, with moderate and then severe CFS/ME participant groups displayed significantly worsened scores respectively (Table 1). There were no statistically significant differences between moderate and severe CFS/ME patient groups years since CFS/ME diagnosis, estimated visits to a general practitioner in the past 12 months or number of days in the past 30 days where symptoms presented difficulties for the patient (Table 1). Severe CFS/ME patients had a significantly increased number of days out of the 30 days prior to participation where 'usual' activities were not able to be carried out due to symptoms (Table 1).

There were no statistically significant differences in blood pressure, pulse, temperature or routine full blood count parameters between participants groups (Table 2).

\section{No significant differences to immunoglobulins}

Serum immunoglobulins, IgA, IgM, IgG2, IgG4 and $\mathrm{IgG}_{\mathrm{Total}}$ were not significantly different between the control, moderate and severe CFS/ME groups (data not shown).

\section{Cytokine Analyses}

IL-1 $\beta$ was correlated with IL-6 according to Spearman's Bivariate Correlation where $p<0.01$. There was a significant increase in IL-1 $\beta$ in moderate CFS/ME compared with severe CFS/ME $(p=0.002)$ (Figure 1) and IL-6 was also significantly decreased in the moderate CFS/ME group compared with the healthy controls and severe CFS/ME group $(p<0.001$ and 0.001 respectively). IL-7 and IL-8 cytokines were significantly increased in the severe CFS/ME group compared with healthy controls and moderate CFS/ME $(p<0.001,0.001$ and $p=0.001,0.001$ respectively) (Figure 1). IL-7 was positively correlated with IFN- $\gamma(p<0.01)$ and IFN- $\gamma$ was significantly increased in severe CFS/ME compared with the moderately affected CFS/ME group $(p=0.025)$ (Figure 2). RANTES was significantly increased in moderate CFS/ME compared with healthy controls and severe CFS/ME ( $p=0.009$ and 0.012 respectively) (Figure 2).

There was no statistical significance found between any of the groups in the cytokines IL-1ra, IL-2, IL-4, IL-5, IL-6, IL-9, IL-10, IL-12p70, IL-13, IL-17, FGF, eotaxin, G-CSF, GM-CSF, IP-10, PDGF-BB, TNF-a and VEGF (Data not shown).

Table 1: Participant and clinical characteristics for each participant group (control, moderate and severe).

\begin{tabular}{|c|c|c|c|c|}
\hline & Control & $\begin{array}{l}\text { Moderate } \\
\text { CFS/ME } \\
\text { patients }\end{array}$ & $\begin{array}{l}\text { Severe } \\
\text { CFS/ME } \\
\text { patients }\end{array}$ & $\begin{array}{l}p \text { Value between } \\
\text { groups }\end{array}$ \\
\hline \multicolumn{5}{|l|}{ Participant Characteristics } \\
\hline Age in years (mean $\pm S D)$ & $40.14 \pm 11.17$ & $42.09 \pm 12.75$ & $40.21 \pm 6.84$ & 0.598 \\
\hline Gender (Female, Male) & 14,8 & 15,7 & 16,3 & 0.324 \\
\hline \multicolumn{5}{|l|}{ CFS/ME Patient Clinical Data } \\
\hline Years since CFS/ME diagnosis & NA & $9.00 \pm 8.870$ & $13.071 \pm 6.639$ & 0.087 \\
\hline Estimated number of visits to a General Practitioner in the last 12 months & NA & $10.12 \pm 5.264$ & $5.214 \pm 4.263$ & 0.174 \\
\hline Number of days in the past 30 days where symptoms/difficulties were present & NA & $26.31 \pm 8.396$ & $29.50 \pm 1.401$ & 0.100 \\
\hline $\begin{array}{l}\text { Number of days in the past } 30 \text { days where usual activities were not able to be } \\
\text { carried out due to symptoms }\end{array}$ & NA & $7.69 \pm 8.875$ & $14.57 \pm 10.733$ & 0.011 \\
\hline \multicolumn{5}{|l|}{ Activity/Ability Level Rating (0-100\%) } \\
\hline Typical good day & $98.89 \pm 3.333$ & $60.45 \pm 19.390$ & $28.18 \pm 14.013$ & 0.000 \\
\hline Typical bad day & $90.00 \pm 11.180$ & $25.00 \pm 14.720$ & $10.00 \pm 0.000$ & 0.000 \\
\hline Day of participation & $97.78 \pm 6.667$ & $50.45 \pm 19.875$ & $14.55 \pm 5.222$ & 0.000 \\
\hline \multicolumn{5}{|l|}{ FibroFatigue Scale - Symptoms } \\
\hline Fatigue & $0.00 \pm 0.000$ & $3.32+1.912$ & $5.64+0.674$ & 0.000 \\
\hline Memory & $0.11 \pm 0.333$ & $3.00 \pm 1.718$ & $3.00 \pm 1.549$ & 0.000 \\
\hline Concentration & $0.00 \pm 0.000$ & $3.23 \pm 1.572$ & $3.73 \pm 1.348$ & 0.000 \\
\hline Sleep & $0.56 \pm 0.882$ & $3.32 \pm 1.555$ & $4.00 \pm 2.191$ & 0.000 \\
\hline Headaches & $0.00 \pm 0.000$ & $2.45 \pm 1.920$ & $3.36 \pm 1.567$ & 0.000 \\
\hline Pain & $0.00 \pm 0.000$ & $3.32+1.615$ & $4.18+1.471$ & 0.000 \\
\hline Muscle Pain & $0.11 \pm 0.333$ & $3.59 \pm 1.652$ & $4.36 \pm 1.567$ & 0.000 \\
\hline Infection & $0.11 \pm 0.333$ & $2.50 \pm 2.064$ & $3.45 \pm 1.753$ & 0.000 \\
\hline Bowel & $0.11 \pm 0.333$ & $1.86 \pm 1.935$ & $2.91 \pm 2.071$ & 0.000 \\
\hline Autonomic & $0.00 \pm 0.000$ & $3.14 \pm 1.612$ & $2.73 \pm 2.149$ & 0.000 \\
\hline Irritability & $0.00 \pm 0.000$ & $2.36 \pm 1.649$ & $1.55 \pm 1.864$ & 0.000 \\
\hline Sadness & $0.00 \pm 0.000$ & $1.73 \pm 1.778$ & $1.36 \pm 1.690$ & 0.001 \\
\hline \multicolumn{5}{|l|}{ Severity Scales } \\
\hline Fatigue Severity Scale & $10.12 \pm 1.219$ & $52.32 \pm 10.403$ & $59.64 \pm 2.649$ & 0.000 \\
\hline Karnofsky Performance Scale & $100.00 \pm 0.000$ & $72.27 \pm 9.726$ & $52.73 \pm 12.721$ & 0.000 \\
\hline Dr Bells Disablity Scale & $100.00 \pm 0.000$ & $49.09 \pm 21.582$ & $20.00 \pm 10.000$ & 0.000 \\
\hline
\end{tabular}


Table 2: Clinical and full blood count results for healthy controls, moderate CFS/ME and severe CFS/ME participant groups.

\begin{tabular}{|c|c|c|c|c|}
\hline Parameter & Control $(n=22)$ & Moderate $(n=22)$ & Severe $(n=19)$ & $p$ value \\
\hline Blood Pressure (Systolic) & $76.36 \pm 9.41$ & $71.14 \pm 5.33$ & $74.74 \pm 11.96$ & 0.172 \\
\hline Blood Pressure (Diastolic) & $119.32 \pm 18.60$ & $114.32 \pm 14.00$ & $111.32 \pm 14.70$ & 0.325 \\
\hline Pulse bpm & $66.00 \pm 8.10$ & $70.82 \pm 8.73$ & $67.16 \pm 6.80$ & 0.115 \\
\hline Temperature $\left({ }^{\circ} \mathrm{C}\right)$ & $36.43 \pm 0.42$ & $36.33 \pm 0.40$ & $36.54 \pm 0.51$ & 0.323 \\
\hline Haemoglobin $\mathrm{g} / \mathrm{L}$ & $135.38 \pm 14.31$ & $141.81 \pm 14.11$ & $135.12 \pm 13.24$ & 0.640 \\
\hline White Cell Count (x109/L) & $5.97 \pm 1.49$ & $5.68 \pm 0.83$ & $6.5 \pm 1.93$ & 0.325 \\
\hline Platelets $(x 109 / \mathrm{L})$ & $229.25 \pm 43.05$ & $242 \pm 43.79$ & $240 \pm 62.63$ & 0.634 \\
\hline Haematocrit (x10 $/ \mathrm{L})$ & $0.40 \pm 0.03$ & $0.42 \pm 0.04$ & $0.40 \pm 0.03$ & 0.478 \\
\hline Red Cell Count $\left(\times 10^{9} / \mathrm{L}\right)$ & $4.54 \pm 0.38$ & $4.70 \pm 0.48$ & $4.44 \pm 0.46$ & 0.088 \\
\hline $\operatorname{MCV}(\mathrm{fL})$ & $88.38 \pm 4.59$ & $88.94 \pm 2.98$ & $91.12 \pm 4.99$ & 0.363 \\
\hline Neutrophils (x109/L) & $3.51 \pm 1.13$ & $3.13 \pm 0.63$ & $3.84 \pm 1.14$ & 0.197 \\
\hline Lymphocytes (x109/L) & $1.96 \pm 0.67$ & $2.01 \pm 0.46$ & $2.09 \pm 0.90$ & 0.907 \\
\hline Monocytes (x109/L) & $0.39 \pm 0.12$ & $0.34 \pm 0.09$ & $0.39 \pm 0.13$ & 0.560 \\
\hline Eosinophils $\left(\times 10^{9} / \mathrm{L}\right)$ & $0.10 \pm 0.06$ & $0.17 \pm 0.09$ & $0.16 \pm 0.12$ & 0.519 \\
\hline Basophils (x109/L) & $0.02 \pm 0.01$ & $0.03 \pm 0.00$ & $0.02 \pm 0.00$ & 0.279 \\
\hline $\mathrm{ESR}(\mathrm{mm} / \mathrm{Hr})$ & $7.06 \pm 2.82$ & $6.13 \pm 5.32$ & $10.06 \pm 5.15$ & 0.056 \\
\hline Sodium (mmol/L) & $138.62 \pm 1.59$ & $138.63 \pm 1.90$ & $138.29 \pm 2.11$ & 0.990 \\
\hline
\end{tabular}

All data is represented as Mean+SD in control ( $\mathrm{n}=22)$, moderate CFS/ME ( $\mathrm{n}=22)$ and severe CFS/ME $(\mathrm{n}=19)$. ${ }^{*}$ signifies $p<0.05$ between participant groups. CFS/ME:

Chronic Fatigue Syndrome/Myalgic Encephalomyelitis; SD: Standard Deviation.

A

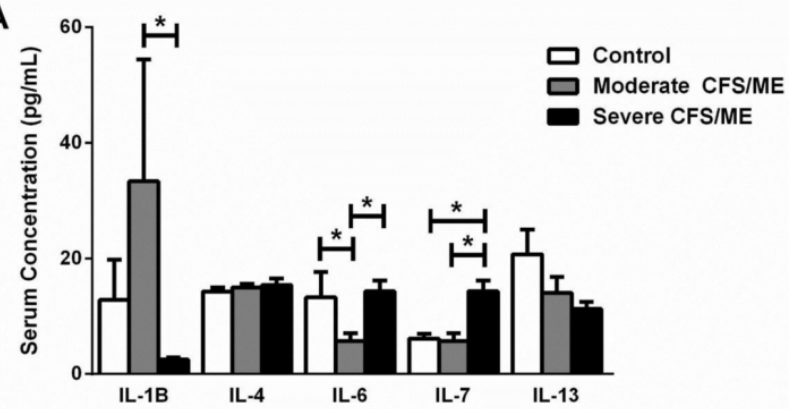

B

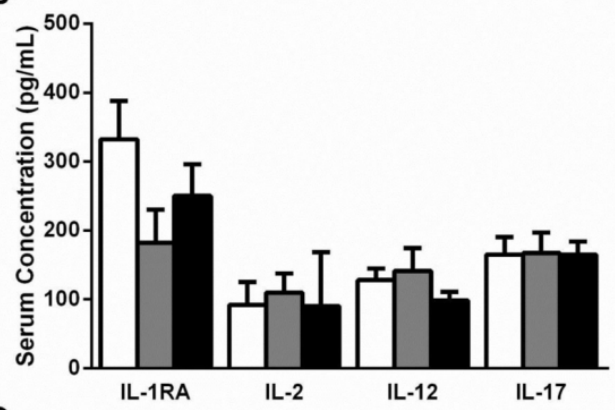

C

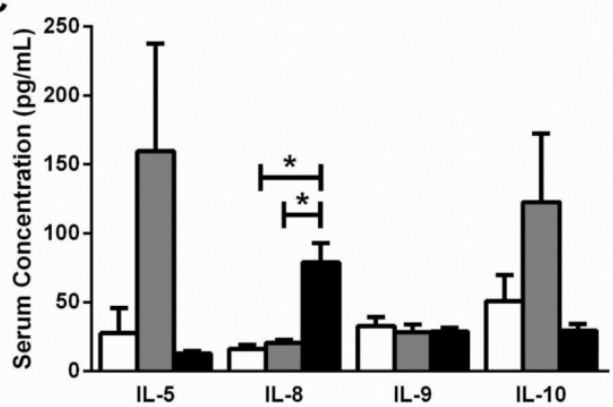

Figure 1: The profile of serum interleukin levels in control, moderate and severe CFS/ME. A IL-1 $\beta$, IL-4, IL-6, IL-7 and IL-13 serum concentrations where data is presented as serum concentration (pg/mL). B IL-1RA, IL-2, IL-12 and IL-17 serum concentrations where data is presented as serum concentration $(\mathrm{pg} / \mathrm{mL})$. C IL-5, IL-8, IL-9 and IL-10 serum concentrations where data is presented as serum concentration $(\mathrm{pg} / \mathrm{mL})$. All data is represented as mean+SEM. * represents results that were significantly different where $p<0.05$. CFS/ME: Chronic Fatigue Syndrome/Myalgic Encephalomyelitis; IL: Interleukin; IL-IRA: interleukin 1 receptor agonist; SEM: Standard Error of the Mean.

\section{Discussion}

This research was the first to assess immunoglobulins and inflammatory cytokines in severe CFS/ME patients compared with moderate CFS/ME patients and healthy controls. There were statistically significant differences in symptom severity and physical activities between controls, moderate and severe CFS/ME patients. According to severity scales, those in the severe CFS/ME patient group displayed the most severe symptoms. Prior to this research, cytokine abnormalities and inconsistently altered immunoglobulin concentrations have been commonly found in both plasma and serum studies of CFS/ME [15, 18-21]. However, literature had only assessed CFS/ME patients who were moderately affected by symptoms, leaving out those who are severely affected and subsequently housebound.

Cytokines and chemokines play a major role in many inflammatory diseases [30, 31]. NK cell cytotoxic activity reflects a balance between activating and inhibitory signals which may be disturbed in CFS/ME patients as they demonstrate consistently reduced NK cell cytotoxic activity compared to healthy controls [2, $9,32,33]$. NK cell activation is triggered by inflammatory mediators, cytokines and chemokines including IL-2, IL-12, IL-15, IL-18 and RANTES following recognition of stressed cells which leads NK cells to lyse target cells and secrete IFN-ץ and TNF-a [31]. NK cells also have an influence on the generation of Th1 type pro-inflammatory responses, which consequently can result in a shift in the Th1/Th2 balance of cytokines. Previously, CFS/ME patients have shown evidence of a bias towards a Th2 immune response as IFN- $\gamma$ and IL-4 were increased in CFS/ME patients compared with healthy controls [20]. 
A

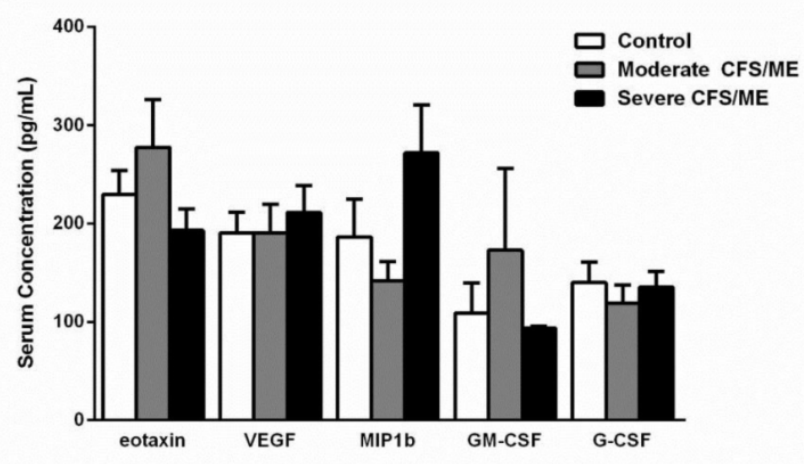

B

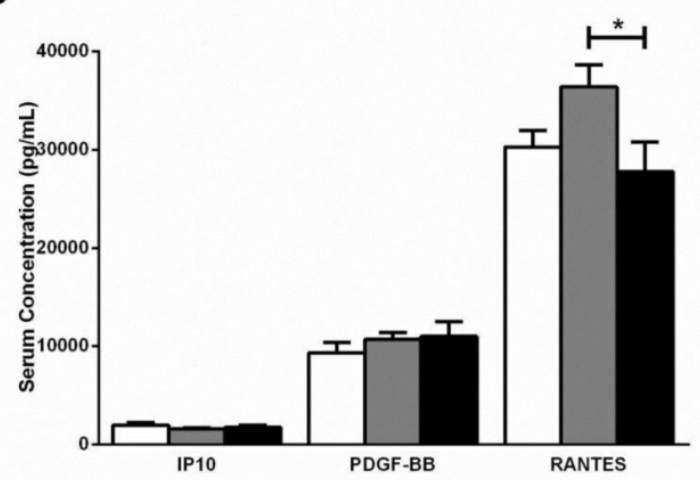

C

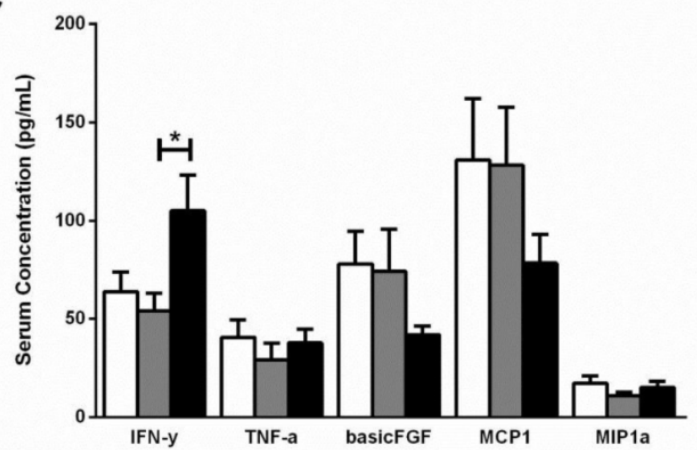

Figure 2: Serum cytokine levels in control, moderate and severe CFS/ME participant groups. A Eotaxin, VEGF, MIPIb, GM-CSF and G-CSF serum concentrations where data is presented as serum concentration $(\mathrm{pg} / \mathrm{mL})$. B IP10, PDGF-BB and RANTES serum concentrations where data is presented as serum concentration ( $\mathrm{pg} / \mathrm{mL})$. C IFN- $\gamma$, TNF- $\alpha$, FGF, MCPI and MIPla serum concentrations where data is presented as serum concentration $(\mathrm{pg} / \mathrm{mL})$. Data are represented as mean+SEM. * represents results that were significantly different where $p<0.05$. CFS/ME: Chronic Fatigue Syndrome/Myalgic Encephalomyelitis; VEGF: vascular endothelial growth factor; MIPIb: macrophage inflammatory protein beta; GM-CSF: granulocyte macrophage colony-stimulating factor; G-CSF: granulocyte colony-stimulating factor; IP10: interferon gamma-induced protein 10; PDGF-BB: platelet-derived growth factor-BB; RANTES: regulated on activation, normal $T$ cell expressed and secreted; IFN-Y: interferon gamma; TNF- $\alpha$ : tumor necrosis factor alpha; FGF: basic fibroblast growth factor; MCP1: monocyte chemotactic protein 1; MIPIa: macrophage inflammatory protein alpha; SEM: Standard Error of the Mean.

IFN-Y is secreted by T cells to form part of a Th1 immune response. The levels of IFN- $\gamma$ in CFS/ME patients are inconsistent, with previous studies demonstrating a reduction of IFN-ץ following mitogenic stimulation of peripheral blood mononuclear cells in the illness [2,34], no difference in the levels of circulating plasma IFN- $\gamma$ [34-36] and increased production of IFN- $\gamma$ by $\mathrm{CD}^{+}$and $\mathrm{CD}^{+} \mathrm{T}$ cells [20]. NK-derived IFN- $\gamma$ leads to the promotion of DC maturation and enhanced antigen presentation to $\mathrm{T}$ cells, hence improving or dampening $\mathrm{T}$ cell responses through IFN- $\gamma[31,33]$. High amounts of IFN- $\gamma$ are secreted by CD56 bright NK cells which exhibit weak cytotoxic activity [33]. Previously, plasma IFN- $\gamma$ levels have significantly differed between CFS/ME patients based on illness duration, with increased plasma IFN- $\gamma$ in patients who had a short illness duration (less than 3 years) [28]. The present study highlights that defining severity of CFS/ME may be important as the increase in serum IFN- $\gamma$ in severe CFS/ME patients may be as a result of overactive IFN- $\gamma$ by CD56 bright $\mathrm{NK}$ cells as a mechanism to enhance the reduced NK cell cytotoxic activity in the illness [8]. This increased serum IFN- $\gamma$ in severe CFS/ME patients may also be contributing to increased DC phenotypes, particularly CD14-CD16 ${ }^{+}$DCs which were increased in severe CFS/ME patients previously [9]. Similarly, increased serum IFN- $\gamma$ may promote heightened $\mathrm{T}$ cell responses, such as the increase in Tregs that is shown in CFS/ME patients [37].

IL-1 $\beta$ is secreted by monocytes and tissue macrophages induced by Th1 cells and functions to regulate metabolic, immuno-inflammatory and reparative properties and it can be a mediator of some diseases $[30,38]$. IL-1 $\beta$ gene polymorphisms may be a marker for the severity of joint destruction in rheumatoid arthritis (RA) [39] as well as a determinant of disease course and severity in patients with inflammatory bowel disease, essentially contributing to the heterogeneity of the illness [40]. IL-1 $\beta$ is involved in the activation of T cells [30], a mechanism which is potentially defective in CFS/ME patients due to the suppression of early $\mathrm{T}$ cell activation and proliferation, possibly also contributing to the lowered inflammatory response in the illness [41]. Increased serum IL-1 $\beta$ in the moderate CFS/ME patients compared with severe CFS/ME patients suggests that patients experiencing moderate symptoms may have increased $\mathrm{T}$ cell activation as a result of enhanced serum IL-1 $\beta$ compared with those who are more severely affected. Interestingly, severe CFS/ME patients' serum IL-1 $\beta$ did not significantly differ from healthy controls, suggesting that in the case of severe CFS/ME, IL- $1 \beta$ is not contributing to any deficiencies in $\mathrm{T}$ cell activation seen in CFS/ME. A previous study demonstrated significantly increased plasma IL-1 $\beta$ in CFS/ME patients with short illness duration (less than 3 years) when compared to CFS/ME patients with a greater illness duration [28]. The present study focused on illness severity, where increased cytokine abnormali- 
ties were reported in IFN- $\gamma$ and IL-1 $\beta$, suggesting that illness severity and duration may play a role in cytokine profiles in CFS/ME.

IL-6 is part of the Th2 immune response and it synergizes with IL-1 in inflammatory reactions and may exacerbate the IL-1 $\beta$ alterations demonstrated in CFS/ME [19]. There was a significant correlation between IL-6 and IL-1 $\beta$ in this study. Increases in IL-1 $\beta$ and IL- 6 in the brain have been linked to 'sickness behaviours' that are exacerbated by pro-inflammatory cytokines and may be present in CFS/ME [21, 42].

IL-6 is a pro-inflammatory cytokine produced by monocytes, epithelial cells and fibroblasts. IL-6 plays a role in the regulation of immune responses, inducing cell proliferation and differentiation, promoting $\mathrm{B}$ cell activation and autoantibody production via $\mathrm{T}$ cell activation [30]. Abnormal or overproduction of IL-6 has also been associated with some autoimmune diseases, such as MS, RA and experimental allergic encephalomyelitis (EAE) [30, 43-45]. Increased IL-6 has been found in CFS/ME patients [35, 36], as well as elevated soluble IL-6 receptor (sIL-6R) which directly enhances the effects of IL-6 [46]. IL-6 is often linked with inflammation or increased inflammatory response [43] and acute infection is associated with elevated plasma concentrations of TNF, IL-6 and IL-8 [43]. Increased IL-6 in severe CFS/ME patients suggests that these patients may have an enhanced inflammatory response compared to those who have less severe symptoms. It has been speculated that increased IL-6 may influence the JAK-STAT3 signalling pathway or the molecular mechanism by which IL-6 regulates the $\mathrm{Na}^{+} / \mathrm{K}^{+}$ATPase, suggesting that it may be beneficial to examine these pathways in CFS/ME patients [45].

Chemokines are the largest family of cytokines and are critical to coordinating adaptive immune responses by binding to specific cell-surface receptors [33]. IL-8 (also known as CXCL8) is a chemokine and an important component of the pro-inflammatory immune response Following activation, NK cells also produce increased amounts of IL- 8 which may lead to the attraction of T cells, B cells and other NK cells [31, 33]. Increased serum IL-8 in severe CFS/ME patients in this research suggests that these patients may be experiencing a heightened pro-inflammatory immune response. Theoretically, increased IL-8 in severely affected CFS/ME patients may demonstrate increased $\mathrm{NK}, \mathrm{T}$ and $\mathrm{B}$ cell recruitment although in CFS/ME, NK cells appear dysfunctional and there is often an imbalance in the activation of these cells $[5,9,32,41]$.

IL-7 can also stimulate cytotoxic functioning in mature peripheral $\mathrm{CD}^{+} \mathrm{T}$ cells, a function that appears to be reduced in CFS/ME patients [47]. An increased serum IL-7 level in the severely affected
CFS/ME patients may be related to an increase in $\mathrm{T}$ cell functioning or NK cell proliferation in the illness. $\mathrm{T}$ cell activation and cytotoxic activity have not been assessed in severe CFS/ME although moderate CFS/ME patients have previously demonstrated reduced numbers of $\mathrm{T}$ cells, including both $\mathrm{CD}^{+}$and $\mathrm{CD}^{+}$subsets as well as decreased $\mathrm{CD} 8^{+} \mathrm{T}$ cell activation [48, 49]. Increased IL-7 should promote T cell function and NK cell proliferation in CFS/ME although both $\mathrm{NK}$ and $\mathrm{T}$ cell function have been reduced in the illness [48, 49]. Therefore, it is possible that there is a defect downstream in the IL-7 pathway that prevents NK and T cell mechanisms in CFS/ME patients. IL-7 was also significantly and positively correlated to IFN- $\gamma$ levels in the present study. Like IFN- $\gamma$, increased IL-7 in severely affected CFS/ME patients may be a response to the reduced NK cell cytotoxic activity typically associated with the illness [9], particularly because NK cell cytotoxic activity appears to be further reduced in severe CFS/ME patients who have higher levels of IFN- $\gamma$. It is also of interest that severe CFS/ME patients demonstrate increases in IFN- $\gamma$ and IL-7, suggesting they may be involved in illness severity, as reported in other illnesses (Dengue infection) [45, 50].

The present study found increased serum RANTES in moderate CFS/ME patients and reduced serum RANTES in severely affected CFS/ME patients. These findings may reflect patient diversity and heterogeneity in the illness. RANTES (or CCL5), is another inflammatory chemokine produced spontaneously by NK cells. RANTES is associated with lymphoid homing, activation of $\mathrm{T}$ cells and their apoptosis as well as resting migration, killing abilities and cytotoxic granule release by NK cells [31, 33, 49, 51-54].

Contrarily, the reduction in RANTES demonstrated in the severely affected CFS/ME patients may suggest a decreased $\mathrm{T}$ cell activation, NK cell lysing abilities and granule release, as shown in previous studies in moderate CFS/ME patients [2, 6, 9, 32]. These findings support previous studies which demonstrate further reduced NK cell cytotoxic activity in severe CFS/ME patients and perhaps highlights a link between some components of the immune response and the symptom presentation and severity experienced by patients $[9,55]$.

In the current study, cytokine levels were marginally above those typically observed in human serum, however as CFS/ME is characterised by immune dysfunction, these elevated levels may represent illness severity presentation. The serum cytokine levels have not impacted the study as the statistical analysis conducted was used to identify differences between the groups. It is also acknowledged that some 
over-the-counter medications or vitamins were being taken by some participants at the time of this research and future studies with larger sample sizes may allow an analysis of specific medications or include a washout period so results are free from the influence of medication.

Further studies into severity subgroups in CFS/ME may be important to explore the mechanisms behind such cytokine alterations. These cytokine changes in CFS/ME may be an indication of further immune dysregulation and the illness pathomechanism.

\section{Conclusions}

The present study is the first to assess serum immunoglobulin and cytokine levels in CFS/ME patients grouped according to symptom severity. All significant serum cytokine differences shown were between moderate and severe CFS/ME patient groups. Interestingly, the moderate CFS/ME patient cohort displayed more extreme levels of some cytokines than the severe CFS/ME patient cohort, which did not significantly differ from healthy controls in IL-1, IL-6, IFN-y and RANTES. On the other hand, the most severe clinical group had distinctively raised levels of IFN- $\gamma$, IL-7 and IL-8. Cytokine abnormalities have been associated with a number of diseases, particularly regarding severity and suggestive pathomechanism, highlighting the importance of the outcomes from the present study and the necessity for future expansions of this research.

Overall, the present study has demonstrated serum cytokine abnormalities in CFS/ME patients. These findings support the investigation of CFS/ME patient severity subgroups in both research and clinical settings. Further identification of different clinical groups in CFS/ME may influence diagnosis and development of therapeutic strategies in future.

\section{Abbreviations}

ADCC: antibody-dependent cell-mediated cytotoxicity; ANOVA: analysis of variance test; CFS/ME: Chronic Fatigue Syndrome/Myalgic Encephalomyelitis; DC: dendritic cell; EAE: experimental allergic encephalomyelitis; FGF: fibroblast growth factor; FSS: fatigue severity scale; G-CSF: granulocyte colony-stimulating factor; GM-CSF: granulocyte macrophage colony-stimulating factor; IFN-ү: interferon gamma; IL-1ra: interleukin 1 receptor agonist; IL-1 $\beta$ : interleukin 1 beta; IQ: interquartile range; IVIg: intravenous immunoglobulin; KPS: karnofsky performance scale; LSD: least significant difference; MS: Multiple Sclerosis; NK: natural killer; PDGF-BB: platelet-derived growth factor-BB; RA: rheumatoid arthritis; RANTES: regulated on activa- tion, normal $\mathrm{T}$ cell expressed and secreted; sIL-6R: elevated soluble IL-6 receptor; SD: standard deviation; SEM: standard error of the mean; SST: serum separating tubes; TNF-a: tumor necrosis factor alpha; VEGF: vascular endothelial growth factor.

\section{Acknowledgements}

Alison Hunter Memorial Foundation, Mason Foundation [Grant Number MA43120] and Queensland Government Department of Science, Information Technology, Innovation and the Arts Smart Futures Fund [Grant Number 216702MRE].

\section{Competing Interest}

The authors have declared that no competing interest exists. The authors alone are responsible for the content and writing of the paper.

\section{References}

1. Pace CN, Trevino S, Prabhakaran E, Scholtz JM. Protein structure, stability and solubility in water and other solvents. Philosophical Transactions of the Royal Society of London Series B: Biological Sciences. 2004; 359: 1225-35.

2. Klimas NG, Salvato FR, Morgan R, Fletcher MA. Immunologic abnormalities in chronic fatigue syndrome. Journal of clinical microbiology. 1990; 28: 1403-10.

3. Carruthers BM, van de Sande MI, De Meirleir KL, Klimas NG, Broderick G, Mitchell T, et al. Myalgic encephalomyelitis: international consensus criteria. Journal of internal medicine. 2011; 270: 327-38.

4. Fukuda K, Straus SE, Hickie I, Sharpe MC, Dobbins JG, Komaroff A. The chronic fatigue syndrome: a comprehensive approach to its definition and study. Annals of internal medicine. 1994; 121: 953-9.

5. Barker E, Fujimura SF, Fadem MB, Landay AL, Levy JA. Immunologic abnormalities associated with chronic fatigue syndrome. Clinical Infectious Diseases. 1994; 18: S136-S41.

6. Brenu EW, van Driel ML, Staines DR, Ashton KJ, Ramos SB, Keane J, et al. Immunological abnormalities as potential biomarkers in chronic fatigue syndrome/myalgic encephalomyelitis. J Transl Med. 2011; 9: 81.

7. Ngonga GK, Ricevuti G. Immunological aspects of chronic fatigue syndrome. Autoimmun Rev. 2009 Feb;8(4):287-91.

8. Patarca-Montero R, Antoni M, Fletcher MA, Klimas NG. Cytokine and other immunologic markers in chronic fatigue syndrome and their relation to neuropsychological factors. Applied Neuropsychology. 2001; 8: 51-64.

9. Hardcastle S, Brenu E, Johnston S, Nguyen T, Huth T, Kaur M. Analysis of the relationship between immune dysfunction and symptom severity in patients with Chronic Fatigue Syndrome/Myalgic Encephalomyelitis (CFS/ME). J Clin Cell Immunol. 2014; 5: 2.

10. Fluge $\varnothing$, Bruland O, Risa K, Storstein A, Kristoffersen EK, Sapkota D, et al. Benefit from B-lymphocyte depletion using the anti-CD20 antibody rituximab in chronic fatigue syndrome. A double-blind and placebo-controlled study. PloS one. 2011; 6: e26358

11. Harrington LE, Mangan PR, Weaver CT. Expanding the effector CD4 T-cell repertoire: the Th17 lineage. Current opinion in immunology. 2006; 18: 349-56.

12. Ziccardi P, Nappo F, Giugliano G, Esposito K, Marfella R, Cioffi M, et al. Reduction of inflammatory cytokine concentrations and improvement of endothelial functions in obese women after weight loss over one year. Circulation. 2002; 105: 804-9.

13. McAfoose J, Baune BT. Evidence for a cytokine model of cognitive function. Neuroscience \& Biobehavioral Reviews. 2009; 33: 355-66.

14. Hill GE, Whitten CW, Landers DF. The influence of cardiopulmonary bypass on cytokines and cell-cell communication. Journal of cardiothoracic and vascular anesthesia. 1997; 11: 367-75.

15. Fletcher MA, Zeng XR, Barnes Z, Levis S, Klimas NG. Plasma cytokines in women with chronic fatigue syndrome. J Transl Med. 2009; 7: 96.

16. Lyall M, Peakman M, Wessely S. A systematic review and critical evaluation of the immunology of chronic fatigue syndrome. Journal of psychosomatic research. 2003; 55: 79-90.

17. Natelson $\mathrm{BH}$, Haghighi MH, Ponzio NM. Evidence for the presence of immune dysfunction in chronic fatigue syndrome. Clinical and diagnostic laboratory immunology. 2002; 9: 747-52.

18. Chao CC, Janoff EN, Hu S, Thomas K, Gallagher M, Tsang M, et al. Altered cytokine release in peripheral blood mononuclear cell cultures from patients with the chronic fatigue syndrome. Cytokine. 1991; 3: 292-8.

19. Patarca R. Cytokines and chronic fatigue syndrome. Annals of the New York Academy of Sciences. 2001; 933: 185-200. 
20. Skowera A, Cleare A, Blair D, Bevis L, Wessely S, Peakman M. High levels of type 2 cytokine-producing cells in chronic fatigue syndrome. Clinical \& Experimental Immunology. 2004; 135: 294-302.

21. Broderick G, Fuite J, Kreitz A, Vernon SD, Klimas N, Fletcher MA. A formal analysis of cytokine networks in chronic fatigue syndrome. Brain, behavior, and immunity. 2010; 24: 1209-17.

22. Lauwerys BR, Garot N, Renauld J-C, Houssiau FA. Cytokine production and killer activity of NK/T-NK cells derived with IL-2, IL-15, or the combination of IL-12 and IL-18. The Journal of Immunology. 2000; 165: 1847-53.

23. Maes M, Twisk FN, Kubera M, Ringel K. Evidence for inflammation and activation of cell-mediated immunity in myalgic encephalomyelitis/chronic fatigue syndrome (ME/CFS): increased interleukin-1, tumor necrosis factor- $\alpha$, PMN-elastase, lysozyme and neopterin. Journal of affective disorders. 2012; 136: 933-9.

24. Neuberger MS. Antibody diversification by somatic mutation: from Burnet onwards. Immunology and cell biology. 2008; 86: 124-32.

25. Schroeder Jr HW, Cavacini L. Structure and function of immunoglobulins. Journal of Allergy and Clinical Immunology. 2010; 125: S41-S52.

26. Tha-In T, Bayry J, Metselaar HJ, Kaveri SV, Kwekkeboom J. Modulation of the cellular immune system by intravenous immunoglobulin. Trends in immunology. 2008; 29: 608-15.

27. Kwak J, Kwak F, Ainbinder S, Ruiz A, Beer A. Elevated peripheral blood natural killer cells are effectively downregulated by immunoglobulin $G$ infusion in women with recurrent spontaneous abortions. American Journal of Reproductive Immunology. 1996; 35: 363-9.

28. Hornig M, Montoya JG, Klimas NG, Levine S, Felsenstein D, Bateman L, et al. Distinct plasma immune signatures in ME/CFS are present early in the course of illness. Science Advances. 2015; 1: e1400121.

29. Aguinis H, Gottfredson RK, Joo H. Best-Practice Recommendations for Defining, Identifying, and Handling Outliers. Organizational Research Methods. 2013; 16: 270-301.

30. Evereklioglu C, Er H, Türköz Y, Çekmen M. Serum levels of TNF-a, sIL-2R, IL-6, and IL-8 are increased and associated with elevated lipid peroxidation in patients with Behçet's disease. Mediators of inflammation. 2002; 11: 87-93.

31. Vivier E, Raulet DH, Moretta A, Caligiuri MA, Zitvogel L, Lanier LL, et al. Innate or adaptive immunity? The example of natural killer cells. Science. 2011; 331: 44-9.

32. Brenu EW, van Driel ML, Staines DR, Ashton KJ, Hardcastle SL, Keane J, et al. Longitudinal investigation of natural killer cells and cytokines in chronic fatigue syndrome/myalgic encephalomyelitis. J Transl Med. 2012; 10: 88.

33. Robertson MJ. Role of chemokines in the biology of natural killer cells. Journal of leukocyte biology. 2002; 71: 173-83.

34. Visser J, Blauw B, Hinloopen B, Brommer E, de Kloet ER, Kluft C, et al. CD4 T lymphocytes from patients with chronic fatigue syndrome have decreased interferon- $\gamma$ production and increased sensitivity to dexamethasone. Journal of Infectious Diseases. 1998; 177: 451-4.

35. Linde A, Andersson B, Svenson SB, Ahrne H, Carlsson M, Forsberg P, et al. Serum levels of lymphokines and soluble cellular receptors in primary Epstein-Barr virus infection and in patients with chronic fatigue syndrome. Journal of Infectious Diseases. 1992; 165: 994-1000.

36. Peakman M, Deale A, Field R, Mahalingam M, Wessely S. Clinical improvement in chronic fatigue syndrome is not associated with lymphocyte subsets of function or activation. Clinical immunology and immunopathology. 1997; 82: 83-91.

37. Brenu E, Johnston $\mathrm{S}$, Hardcastle $\mathrm{S}$, Huth $\mathrm{T}$, Fuller $\mathrm{K}$, Ramos $\mathrm{S}$, et al. Immune abnormalities in patients meeting new diagnostic criteria for chronic fatigue syndrome/Myalgic Encephalomyelitis. J Mol Biomark Diagn. 2013; 4: 4172.

38. Chizzolini C, Chicheportiche R, Burger D, Dayer JM. Human Th1 cells preferentially induce interleukin (IL)-1beta while Th2 cells induce IL-1 receptor antagonist production upon cell/cell contact with monocytes. Eur J Immunol. 1997; 27: 171-7.

39. Buchs N, Di Giovine F, Silvestri T, Vannier E, Duff G, Miossec P. IL-1B and IL-1Ra gene polymorphisms and disease severity in rheumatoid arthritis: interaction with their plasma levels. Genes and immunity. 2001; 2: 222-8.

40. Nemetz A, Nosti-Escanilla MP, Molnár T, Köpe A, Kovács Á, Fehér J, et al. IL1B gene polymorphisms influence the course and severity of inflammatory bowel disease. Immunogenetics. 1999; 49: 527-31.

41. Maes M, Mihaylova I, Leunis J-C. In chronic fatigue syndrome, the decreased levels of omega- 3 poly-unsaturated fatty acids are related to lowered serum zinc and defects in T cell activation. Neuroendocrinology Letters. 2005; 26: 745-51.

42. Vollmer-Conna U, Fazou C, Cameron B, Li H, Brennan C, Luck L, et al. Production of pro-inflammatory cytokines correlates with the symptoms of acute sickness behaviour in humans. Psychological medicine. 2004; 34: 1289-97.

43. Evans CA, Jellis J, Hughes SP, Remick DG, Friedland JS. Tumor necrosis factor- $\alpha$, interleukin- 6 , and interleukin- 8 secretion and the acute-phase response in patients with bacterial and tuberculous osteomyelitis. Journal of Infectious Diseases. 1998; 177: 1582-7.

44. Kimura A, Kishimoto T. IL-6: Regulator of Treg/Th17 balance. European journal of immunology. 2010; 40: 1830-5

45. Ishihara K, Hirano T. IL-6 in autoimmune disease and chronic inflammatory proliferative disease. Cytokine \& growth factor reviews. 2002; 13: 357-68.

46. Patarca R, Klimas NG, Garcia MN, Walters MJ, Dombroski D, Pons $H$, et al. Dysregulated expression of soluble immune mediator receptors in a subset of patients with chronic fatigue syndrome: cross-sectional categorization of patients by immune status. Journal of Chronic Fatigue Syndrome. 1995; 1: 81-96.

47. Maher KJ, Klimas NG, Fletcher MA. Chronic fatigue syndrome is associated with diminished intracellular perforin. Clinical \& Experimental Immunology. 2005; 142: 505-11.

48. Lloyd AR, Wakefield D, Boughton C, Dwyer J. Immunological abnormalities in the chronic fatigue syndrome. The Medical Journal of Australia. 1989; 151: 122.

49. Curriu M, Carrillo J, Massanella M, Rigau J, Alegre J, Puig J, et al. Screening NK-, B-and T-cell phenotype and function in patients suffering from Chronic Fatigue Syndrome. Journal of translational medicine. 2013; 11: 68.

50. Gunther V, Putnak R, Eckels K, Mammen M, Scherer J, Lyons A, et al. A human challenge model for dengue infection reveals a possible protective role for sustained interferon gamma levels during the acute phase of illness. Vaccine. 2011; 29: 3895-904.

51. Jonsson R, Brun JG. Sjögren's syndrome. Wiley Online Library; 2010

52. Brenu EW, Staines DR, Baskurt OK, Ashton KJ, Ramos SB, Christy RM, et al. Immune and hemorheological changes in chronic fatigue syndrome. Journal of translational medicine. 2010; 8: 1 .

53. Tyner JW, Uchida O, Kajiwara N, Kim EY, Patel AC, O'Sullivan MP, et al. CCL5-CCR5 interaction provides antiapoptotic signals for macrophage survival during viral infection. Nature medicine. 2005; 11: 1180-7.

54. Murooka TT, Wong MM, Rahbar R, Majchrzak-Kita B, Proudfoot AE, Fish EN. CCL5-CCR5-mediated Apoptosis in $\mathrm{T}$ Cells REQUIREMENT FOR GLYCOSAMINOGLYCAN BINDING AND CCL5 AGGREGATION. Journal of Biological Chemistry. 2006; 281: 25184-94.

55. Brenu, Hardcastle, Atkinson G, van Driel M, Kreijkamp-Kaspers S, Ashton K, et al. Natural killer cells in patients with severe chronic fatigue syndrome. Autoimmunity Highlights. 2013;: 1-12. 